E2-2005-76

I. Zborovský1 ${ }^{1}$ M. Tokarev²

\title{
GENERALIZED $z$-SCALING FOR CHARGED HADRONS AND JETS
}

Submitted to «Particles and Nuclei, Letters»

\footnotetext{
${ }^{1}$ Nuclear Physics Institute, Academy of Sciences of the Czech Republic, Řež, Czech Republic

${ }^{2}$ E-mail: tokarev@sunhe.jinr.ru
} 
Зборовский И., Токарев М.

Обобщенный $z$-скейлинг для заряженных адронов и струй

Предложено обобщение $z$-скейлинга, установленного ранее для инклюзивного рождения заряженных адронов с большими поперечными импульсами и струй в протон(анти)протонных столкновениях. Построена скейлинговая функция, описывающая оба класса событий в широком диапазоне энергий столкновения и плотностей множественности заряженных частиц, исследованных коллаборациями UA1, E735 и CDF. Определены аномальные фрактальные размерности сталкивающихся и рожденных объектов и параметры, характеризующие среду, ассоциированную с рождением адрона и струи. Полученные результаты для высоких плотностей множественности подтверждают ранее установленные свойства $z$-скейлинга для «minimum bias»-событий. Новые данные о поведении спектров заряженных адронов в протон-протонных взаимодействиях в зависимости от плотности множественности в центральной области, полученные коллаборацией STAR, дают дополнительное подтверждение установленной закономерности. Представляет интерес дальнейшая экспериментальная проверка свойств $z$-скейлинга и его использование для поиска новых явлений при рождении частиц с большими поперечными импульсами и в событиях с высокой множественностью на ускорителях U70, тэватрон, RHIC и LHC.

Работа выполнена в Лаборатории высоких энергий им. В. И. Векслера и А. М. Балдина ОИЯИ.

Препринт Объединенного института ядерных исследований. Дубна, 2005

Zborovský I., Tokarev M.

E2-2005-76

Generalized $z$-Scaling for Charged Hadrons and Jets

Generalization of $z$-scaling observed in the inclusive high- $p_{T}$ charged hadron and jet production is proposed. The scaling function $\psi(z)$ describing both charged hadrons and jets produced in proton-(anti)proton collisions for various multiplicity densities and collision energies is constructed. Anomalous fractal dimensions and parameters characterizing associated medium for both classes of events are established. The basic features of the scaling established in minimum bias events are shown to be preserved up to the highest multiplicity densities measured in experiments UA1, E735, CDF and STAR. The obtained results are of interest to use $z$-scaling as a tool for searching for new physics phenomena of particle production at high transverse momentum and in high-multiplicity region at the U70, Tevatron, RHIC and LHC.

The investigation has been performed at the Veksler and Baldin Laboratory of High Energies, JINR. 


\section{INTRODUCTION}

Collision of hadrons and nuclei at sufficiently high energies is an ensemble of individual interactions of their constituents. The constituents are partons in the parton model or quarks and gluons in the theory of QCD. Production of particles with large transverse momenta from such reactions has relevance to constituent interactions at small scales. This regime is of interest to search for new physics phenomena in elementary processes such as quark compositeness [1], extra dimensions [2], black holes [3], fractal space-time [4], etc. Other aspects of high energy interactions are connected with small momenta of secondary particles and high multiplicities. This regime has relevance to collective phenomena of particle production. Search for new physics in both regions is one of the main goals of investigations at the Relativistic Heavy Ion Collider (RHIC) at BNL and at the Large Hadron Collider (LHC) at CERN. Experimental data of particle production can give constraints for different theoretical models. Processes with high transverse momenta of produced particles are most suitable for precise test of perturbative QCD. The soft regime is of interest for verification of non-perturbative QCD and investigation of phase transitions in non-Abelian theories.

Many approaches to description of particle production are used to search for regularities reflecting general principles at high energies [5-14]. One of the most basic principles is the self-similarity of hadron production valid both in soft and hard physics. Other general principles are locality and fractality which can be applied to hard processes at small scales. The locality of hadronic interactions follows from numerous experimental and theoretical studies. These investigations showed that interactions of hadrons and nuclei can be described in terms of the interactions of their constituents. Fractality in hard processes is specific feature connected with substructure of the constituents. This includes the self-similarity over wide scale range. Fractality of soft processes concerning the multi-particle production was investigated by many authors.

Fractality in inclusive reactions with high- $p_{T}$ particles was considered for the first time in the framework of $z$-scaling [15]. The approach is based on principles of fractality, locality and self-similarity. It takes into account fractal structure of the colliding objects, interaction of their constituents and particle formation. The observed regularity is expressed in terms of the scaling function $\psi(z)$ and the scaling variable $z$ constructed via the experimentally measured inclusive cross section $E d^{3} \sigma / d p^{3}$ and the multiplicity density $d N / d \eta$. Data 
$z$-presentation reveals properties of energy and angular independence and power law at high $z$. The function $\psi(z)$ is interpreted as probability density to produce a particle with formation length $z$.

In the present paper we generalize the concept of $z$-scaling for various multiplicities of produced particles. We show that generalized scaling represents regularity in both soft and hard regimes in proton-(anti)proton collisions over a wide range of multiplicities. Connection of the $z$-scaling with entropy and heat capacity of the colliding system is discussed. The construction is applied to experimental data on inclusive cross sections of charged hadrons and jets. The same scaling function $\psi(z)$ was established to describe production of both classes of events. The analysis of data shows simultaneously that anomalous fractal dimensions and «heat capacity» for charged hadrons and jets are different. This indicates that production mechanism is influenced differently by the system at hadron and parton levels. The self-similarity of the production processes governed by the same law is common to both of them. The obtained results give additional confirmation of self-similarity and fractality in hadronic processes at high energies. We suggest to use $z$-scaling as a tool for searching for new physics phenomena of particle production in high transverse momentum and high multiplicity region at the U70, Tevatron, RHIC and LHC.

\section{1. $z$-SCALING}

We would like to emphasize two main points of our approach [15]. The first one is based on self-similarity of the mechanism underlying particle production on the level of the elementary constituent interactions. The interactions are governed by local energy-momentum conservation law. The second one is fractal character of the parton content of the composite structures involved.

1.1. Locality, Self-Similarity and Fractality. The idea of $z$-scaling is based on the assumption [10] that gross features of inclusive particle distribution of the reaction

$$
M_{1}+M_{2} \rightarrow m_{1}+X
$$

can be described at high energies in terms of the corresponding kinematic characteristics of constituent subprocesses. The subprocess is considered as binary collision

$$
\left(x_{1} M_{1}\right)+\left(x_{2} M_{2}\right) \rightarrow m_{1} / y+\left(x_{1} M_{1}+x_{2} M_{2}+m_{2} / y\right)
$$

of constituents which carry out the fractions $x_{1}$ and $x_{2}$ of the incoming 4-momenta $P_{1}$ and $P_{2}$ of the objects with the masses $M_{1}$ and $M_{2}$. The inclusive particle with the mass $m_{1}$ and the 4-momentum $p$ carries out the fraction 
$y$ of the 4-momentum of the outgoing constituent. The constituent interaction satisfies the energy-momentum conservation written in the form

$$
\left(x_{1} P_{1}+x_{2} P_{2}-p / y\right)^{2}=\left(x_{1} M_{1}+x_{2} M_{2}+m_{2} / y\right)^{2} .
$$

The parameter $m_{2}$ is introduced to satisfy the internal conservation laws (for baryon number, isospin, strangeness, and so on). The equation is the expression of locality of hadron interaction at constituent level.

The constituent interactions are assumed to be similar. This property is connected with dropping of certain dimensional quantities out of description of physical phenomena. The self-similar solutions are constructed in terms of selfsimilarity parameters. We search for the solution

$$
\psi(z)=\frac{1}{N \sigma} \frac{d \sigma}{d z}
$$

depending on single self-similarity parameter $z$. Here $\sigma$ is the inelastic cross section of reaction (1) and $N$ is particle multiplicity. The parameter $z$ is specific dimensionless combination of quantities which characterize particle production in high-energy inclusive reactions. It depends on momenta and masses of the colliding and inclusive particles, multiplicity density and structural characteristics of the interacting objects. We define the self-similarity parameter $z$ in the form

$$
z=z_{0} \Omega^{-1},
$$

where

$$
\Omega\left(x_{1}, x_{2}, y\right)=\left(1-x_{1}\right)^{\delta_{1}}\left(1-x_{2}\right)^{\delta_{2}}(1-y)^{\epsilon} .
$$

The parameter $z$ has a character of a fractal measure. For a given production process, its finite part $z_{0}$ is proportional to the kinetic transverse energy released in the underlying collision of constituents. The divergent part $\Omega^{-1}$ describes the resolution at which the collision of the constituents can be singled out of this process. The $\Omega\left(x_{1}, x_{2}, y\right)$ is relative number of parton configurations containing constituents which carry out the fractions $x_{1}$ and $x_{2}$ of the incoming momenta $P_{1}$ and $P_{2}$ and the outgoing constituent which fraction $y$ is carried out by the inclusive particle with the momentum $p$. The $\delta_{1}, \delta_{2}$ and $\epsilon$ are anomalous fractal dimensions of the incoming and outgoing objects, respectively. Common property of fractal measures is their divergence with the increasing resolution

$$
z(\Omega) \rightarrow \infty \quad \text { if } \quad \Omega^{-1} \rightarrow \infty .
$$

For the infinite resolution, the momentum fractions become unity, $x_{1}=x_{2}=$ $y=1$ and $\Omega=0$. The kinematical limit corresponds to the fractal limit $z=\infty$. 
1.2. Principle of Minimal Resolution and Momentum Fractions. Let us formulate principle of minimal resolution $\Omega^{-1}$ of the fractal measure $z$ with respect to all binary collisions of constituents from which the inclusive particle with the momentum $p$ can be produced. This singles out the underlying subprocess of the constituents with the resolution beyond which there is no sense to consider their substructure. In such a way, the momentum fractions $x_{1}, x_{2}$ and $y$ are determined to minimize $\Omega^{-1}\left(x_{1}, x_{2}, y\right)$ taking into account the energy-momentum conservation in binary collision (2). This is equivalent to the solution of the system of equations

$$
\frac{\partial \Omega\left(x_{1}, x_{2}, y\right)}{\partial x_{1}}=0, \quad \frac{\partial \Omega\left(x_{1}, x_{2}, y\right)}{\partial x_{2}}=0, \quad \frac{\partial \Omega\left(x_{1}, x_{2}, y\right)}{\partial y}=0
$$

with condition (3). The principle of minimal resolution leads to the decomposition of the momentum fractions

$$
x_{1}=\lambda_{1} / y+\chi_{1}(\alpha, y) / y \quad x_{2}=\lambda_{2} / y+\chi_{2}(\alpha, y) / y
$$

at fixed $y$. Using the decomposition, expression (2) can be rewritten in the symbolic form

$$
x_{1}+x_{2} \rightarrow\left(\lambda_{1}+\lambda_{2}\right) / y+\left(\chi_{1}+\chi_{2}\right) / y .
$$

This relation means that $\lambda$-parts of the interacting partons contribute to the production of the inclusive particle, while the $\chi$-parts are responsible for the creation of its recoil. Here we use the following notations:

$$
\chi_{1}=\sqrt{\mu_{1}^{2}+\omega_{1}^{2}}-\omega_{1}, \quad \chi_{2}=\sqrt{\mu_{2}^{2}+\omega_{2}^{2}}+\omega_{2},
$$

where

$$
\mu_{1}^{2}=\left(\lambda_{1} \lambda_{2}+\lambda_{0}\right) \alpha \frac{y-\lambda_{1}}{y-\lambda_{2}}, \quad \mu_{2}^{2}=\left(\lambda_{1} \lambda_{2}+\lambda_{0}\right) \alpha^{-1} \frac{y-\lambda_{2}}{y-\lambda_{1}}
$$

The parameter $\alpha=\delta_{2} / \delta_{1}$ is the ratio of the anomalous fractal dimensions of the colliding objects and

$$
\lambda_{1}=\frac{\left(P_{2} p\right)+M_{2} m_{2}}{\left(P_{1} P_{2}\right)-M_{1} M_{2}}, \quad \lambda_{2}=\frac{\left(P_{1} p\right)+M_{1} m_{2}}{\left(P_{1} P_{2}\right)-M_{1} M_{2}}, \quad \lambda_{0}=\frac{0.5\left(m_{2}^{2}-m_{1}^{2}\right)}{\left(P_{1} P_{2}\right)-M_{1} M_{2}} .
$$

The $\omega_{i}=\mu_{i} U(i=1,2)$ are expressed through the quantity

$$
U=\frac{\alpha-1}{2 \sqrt{\alpha}} \xi
$$


with the kinematical factor

$$
\xi=\sqrt{\frac{\lambda_{1} \lambda_{2}+\lambda_{0}}{\left(y-\lambda_{1}\right)\left(y-\lambda_{2}\right)}},
$$

$(0 \leq \xi \leq 1)$, characterizing scale of constituent interaction. Solution of system (8) with condition (3) can be obtained by iterations of expressions (9) with respect to $y$.

1.3. Scaling Variable $z$. Search for an adequate, physically meaningful but still sufficiently simple form of the self-similarity parameter $z$ plays a crucial role in our approach. We define the scaling variable $z$ in the form

$$
z=\frac{s_{\perp}^{1 / 2}}{\left(d N /\left.d \eta\right|_{0}\right)^{c} \cdot m_{0}} \cdot \Omega^{-1} .
$$

Here $m_{0}$ is the nucleon mass. Minimal transverse kinetic energy of the constituent subprocess is determined by the formula

$$
s_{\perp}^{1 / 2}=s_{\lambda}^{1 / 2}+s_{\chi}^{1 / 2}-m_{1}-\left(M_{1} x_{1} y+M_{2} x_{2} y+m_{2}\right) .
$$

This energy consists of two parts

$$
s_{\lambda}^{1 / 2}=\sqrt{\left(\lambda_{1} P_{1}+\lambda_{2} P_{2}\right)^{2}}, \quad s_{\chi}^{1 / 2}=\sqrt{\left(\chi_{1} P_{1}+\chi_{2} P_{2}\right)^{2}},
$$

which represent the energy for the creation of the inclusive particle and its recoil, respectively. The boundaries of the range of $z$ are 0 and $\infty$. They are accessible at any collision energy and do not depend on the kinematical factor $\xi$.

The $d N /\left.d \eta\right|_{0}$ is multiplicity density at pseudorapidity $\eta=0$. It depends on state of the produced medium in the colliding system. The parameter $c$ characterizes properties of this medium. The quantity

$$
W=\left(d N /\left.d \eta\right|_{0}\right)^{c} \cdot \Omega
$$

is proportional to all parton and hadron configurations of the colliding system resulting to production of the inclusive particle with the momentum $p$. The scaling variable (16) has physical meaning of the ratio

$$
z=\frac{s_{\perp}^{1 / 2}}{W \cdot m_{0}}
$$

of the minimal transverse kinetic energy of the constituent subprocess and the number of the configurations $W$. 
1.4. Scaling Variable $z$ and Entropy $S$. Let us introduce correspondence between the scaling variable and entropy. According to statistical physics, entropy of a system is given by number of all statistical states $W$ of the system as follows:

$$
S=\ln W .
$$

In thermodynamics, entropy for ideal gas is determined by the formula

$$
S=c_{V} \ln T+R \ln V+\text { const. }
$$

The $c_{V}$ is heat capacity and $R$ is universal constant. The temperature $T$ and the volume $V$ characterize state of the system. Using (19) and (21), we can write

$$
S=c \ln \left[d N /\left.d \eta\right|_{0}\right]+\ln \left[\left(1-x_{1}\right)^{\delta_{1}}\left(1-x_{2}\right)^{\delta_{2}}(1-y)^{\epsilon}\right]+\text { const. }
$$

Exploiting analogy between (22) and (23), we interpret the parameter $c$ as «heat capacity» of the medium. The multiplicity density $d N /\left.d \eta\right|_{0}$ has physical meaning of «temperature» of the colliding system. The second term in (23) depends on the volume in space of the momentum fractions $\left\{x_{1}, x_{2}, y\right\}$ containing such constituent configurations which can contribute to production of the inclusive particle with the momentum $p$. On the basis of this analogy we can say that entropy of the colliding system increases with the multiplicity density and decreases with increasing resolution $\Omega^{-1}$.

1.5. Scaling Function $\psi(z)$. The scaling function $\psi(z)$ is expressed in terms of the experimentally measured inclusive invariant cross section $E d^{3} \sigma / d p^{3}$, multiplicity density $d N / d \eta$ and the total inelastic cross section $\sigma_{\text {in }}$. Exploiting definition (4) one can obtain the expression

$$
\psi(z)=-\frac{\pi s}{(d N / d \eta) \sigma_{i n}} J^{-1} E \frac{d^{3} \sigma}{d p^{3}} .
$$

Here, $s$ is the center-of-mass collision energy squared and

$$
J=\frac{\partial y}{\partial \lambda_{1}} \frac{\partial z}{\partial \lambda_{2}}-\frac{\partial y}{\partial \lambda_{2}} \frac{\partial z}{\partial \lambda_{1}}
$$

is the corresponding Jacobian. The rapidity is given by

$$
y=\frac{1}{2} \ln \frac{\lambda_{2}}{\lambda_{1}} .
$$

The function $\psi(z)$ is normalized as follows:

$$
\int_{0}^{\infty} \psi(z) d z=1
$$

The relation allows us to interpret the $\psi(z)$ as a probability density to produce inclusive particle with the corresponding value of the variable $z$. The variable $z$ was interpreted as a particle formation length. 


\section{MULTIPLICITY DEPENDENCE OF $\psi(z)$ FOR CHARGED HADRONS}

We analyze experimental data [16-19] on charge hadron production in $p \bar{p}$ and $p p$ collisions at different multiplicities and energies over a wide- $p_{T}$ range.

The transverse momentum distributions of charged hadrons produced in $p \bar{p}$ collisions were measured by the UA1 Collaboration at the energy $\sqrt{s}=540 \mathrm{GeV}$ for $d N_{\mathrm{ch}} / d \eta=0.8-14.9$ at the $S \bar{p} p S$ [16]. The measurements covered 5 units of pseudorapidity in the central region of the collisions, $|\eta|<2.5$. Multiplicity dependence of the transverse momentum distributions were measured up to $6 \mathrm{GeV} / \mathrm{c}$. Figure 1, a demonstrates strong sensitivity of the spectra to the multiplicity density at high $p_{T}$. The same data are presented in Fig. $1, b$ in the scaling form. The scaling function $\psi(z)$ changes over 6 orders of magnitude in
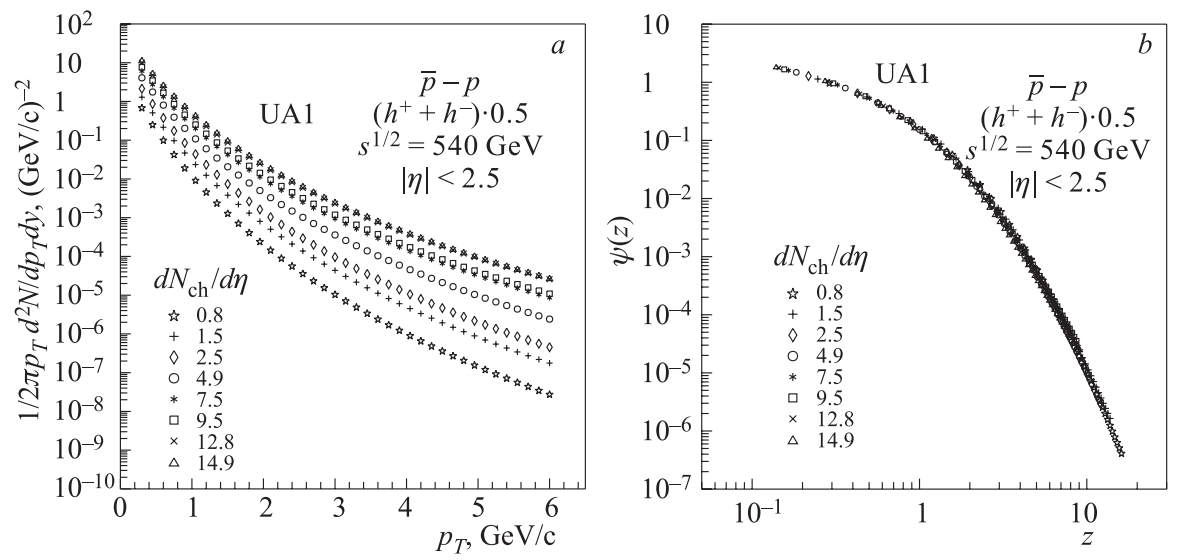

Fig. 1. a) Multiplicity dependence of charged hadron spectra in $\bar{p} p$ collisions at $\sqrt{s}=$ $540 \mathrm{GeV}$. Experimental data are obtained by the UA1 Collaboration [16]. b) The corresponding scaling function $\psi(z)$

the range $z=0.1-15$. The independence of $\psi$ on multiplicity density $d N / d \eta$ is observed. The result gives strong restriction on the parameter $c$. It was found to be $c=0.25$.

The E735 Collaboration measured the multiplicity dependence of charged hadron spectra in proton-antiproton collisions at the energy $\sqrt{s}=1800 \mathrm{GeV}$ for $d N_{\mathrm{ch}} / d \eta=2.3-26.2$ at the Tevatron [17]. This includes measurements at the highest multiplicity density and energy. The pseudorapidity range was $|\eta|<3.25$. Data cover the transverse momentum range $p_{T}=0.15-3 \mathrm{GeV} / \mathrm{c}$. Figure 2, $a$ confirms strong dependence of the spectra on the multiplicity observed at the $S \bar{p} p S$. Figure 2,b shows the same data in the $z$-presentation. The independence of the scaling function $\psi(z)$ was found with the same value $c=0.25$. This includes region of small $z$ up to 0.03 . 

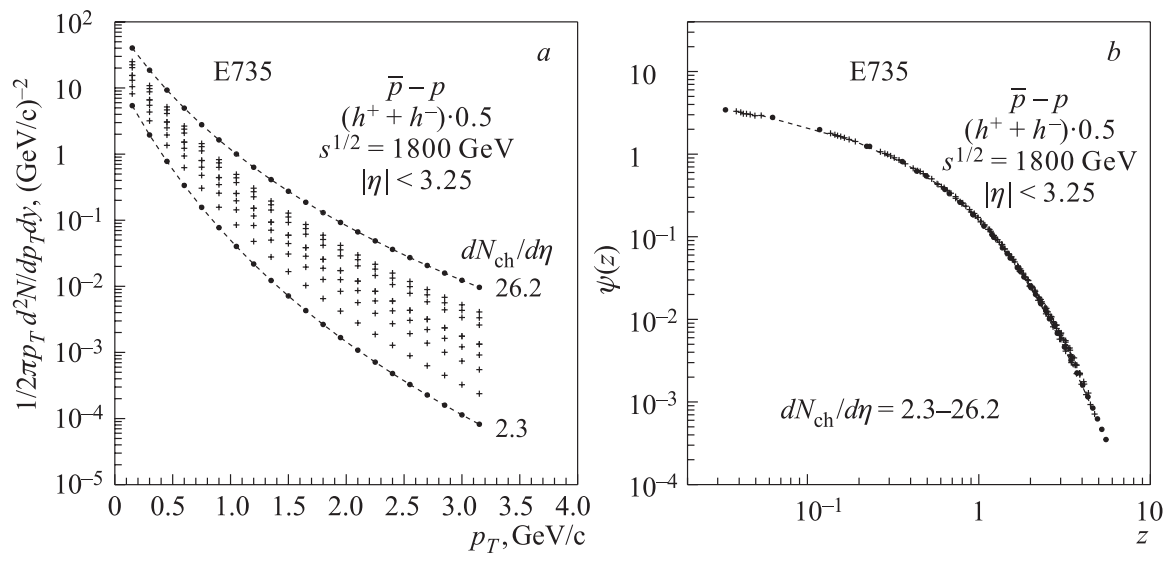

Fig. 2. a) Multiplicity dependence of charged hadron spectra in $\bar{p} p$ collisions at $\sqrt{s}=$ $1800 \mathrm{GeV}$. Experimental data are obtained by the E735 Collaboration [17]. b) The corresponding scaling function $\psi(z)$

New data of the multiplicity dependence on charged hadron spectra were obtained by the CDF Collaboration at the Tevatron [18]. The data were taken at collision energies $\sqrt{s}=630$ and $1800 \mathrm{GeV}$. The particles were registered in the pseudorapidity range $|\eta|<1$. The spectra were measured up to $10 \mathrm{GeV} / \mathrm{c}$. Figure $3, a, b$ shows spectra for multiplicity densities of $2.5,5.0$ and 7.5. The multiplicity dependence of the spectra increases with $p_{T}$. It is additional confirmation of the same effect observed by the UA1 and E735 Collaborations. Both $\mathrm{CDF}$ data sets are plotted in $z$-presentation in Fig. 3, c. The scaling function reveals the energy and multiplicity independence at the same value $c=0.25$. We note that the scaling for charged particle production in proton-antiproton collisions for different multiplicities and energies is consistent with the values of the anomalous fractal dimensions $\delta_{1}=1, \delta_{2}=1$ and $\epsilon=1$.

The STAR Collaboration obtained the new data [19] on the inclusive spectrum of charged hadrons produced in $p p$ collisions in the central rapidity range $|\eta|<0.5$ at the energy $\sqrt{s}=200 \mathrm{GeV}$. The transverse momentum spectra were measured up to $9.5 \mathrm{GeV} / \mathrm{c}$. Figure $4, a$ demonstrates the strong dependence of the spectra on multiplicity density over the range $d N_{\mathrm{ch}} / d \eta=2.5,6.0$ and 8.0. The STAR data for proton-proton collisions at the RHIC confirm the multiplicity independence of the scaling function established for proton-antiproton collisions at higher energies. It was also found that the value of the parameter $c$ is equal to 0.25 .

Thus we can conclude that the available experimental data on the multiplicity dependence of charged particle spectra at various energies in $p \bar{p}$ and $p p$ collisions confirm generalized $z$-scaling for the same value of the parameter $c$. 

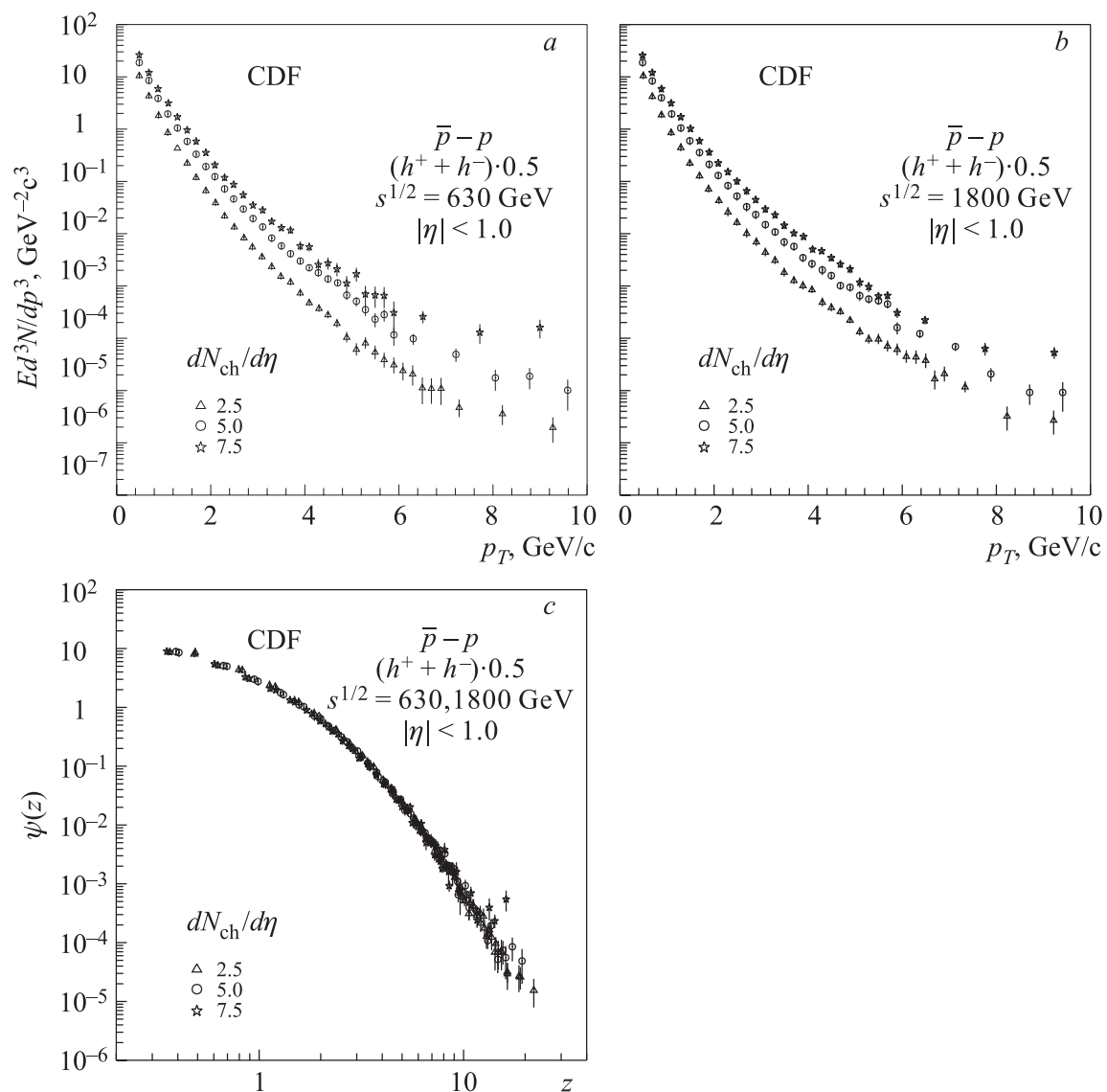

Fig. 3. Multiplicity dependence of charged hadron spectra in $\bar{p} p$ collisions at $\sqrt{s}=630$

(a) and $1800 \mathrm{GeV}(b)$. Experimental data are obtained by the CDF Collaboration [18]. (c) The corresponding scaling function $\psi(z)$

\section{SCALING FUNCTION FOR CHARGED HADRONS AND JETS}

Production of charged hadrons with large transverse momenta has deep correspondence with the jet production. Jets are collimated groups of hadrons including a leading particle with high enough $p_{T}$ and associated hadrons. Jets are considered as direct manifestation of hard parton scattering.

Charged hadron spectra were measured over a wide range of collision energies $\sqrt{s}=200-1800 \mathrm{GeV}$ in proton-antiproton collisions. The transverse momentum range covered $0.2-26 \mathrm{GeV} / \mathrm{c}$. The experimental data on inclusive cross sections 

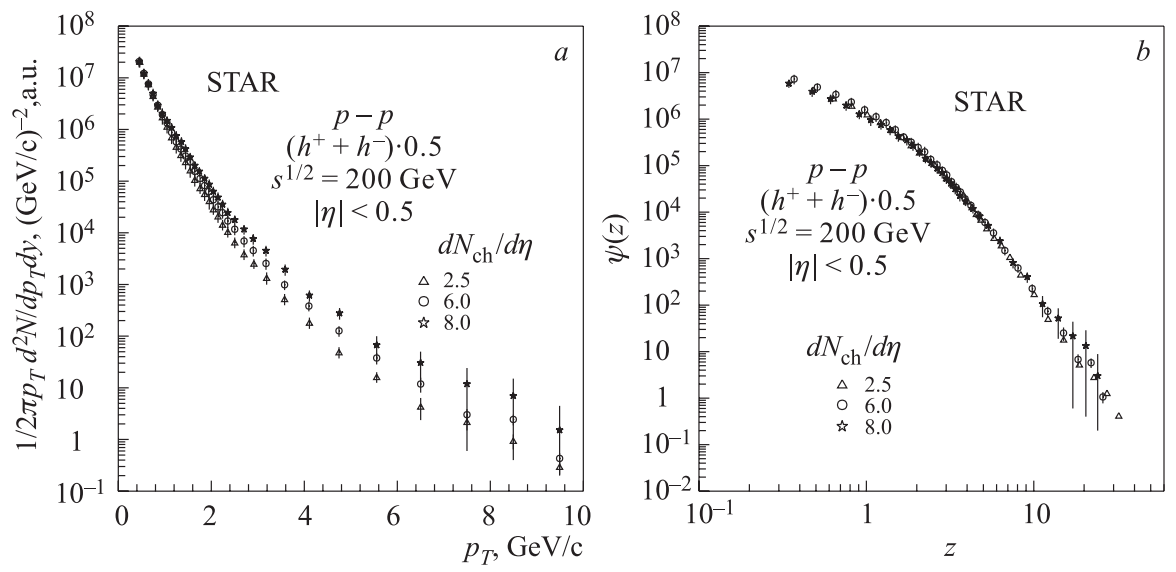

Fig. 4. a) Multiplicity dependence of charged hadron spectra in $p p$ collisions at $\sqrt{s}=$ $200 \mathrm{GeV}$. Experimental data are obtained by the STAR Collaboration [19]. b) The corresponding scaling function $\psi(z)$

obtained at the $S \bar{p} p S$ and Tevatron are shown in Fig. 5, $a$. The cross sections of jets are much smaller. The D0 Collaboration data on jet production at $\sqrt{s}=630$
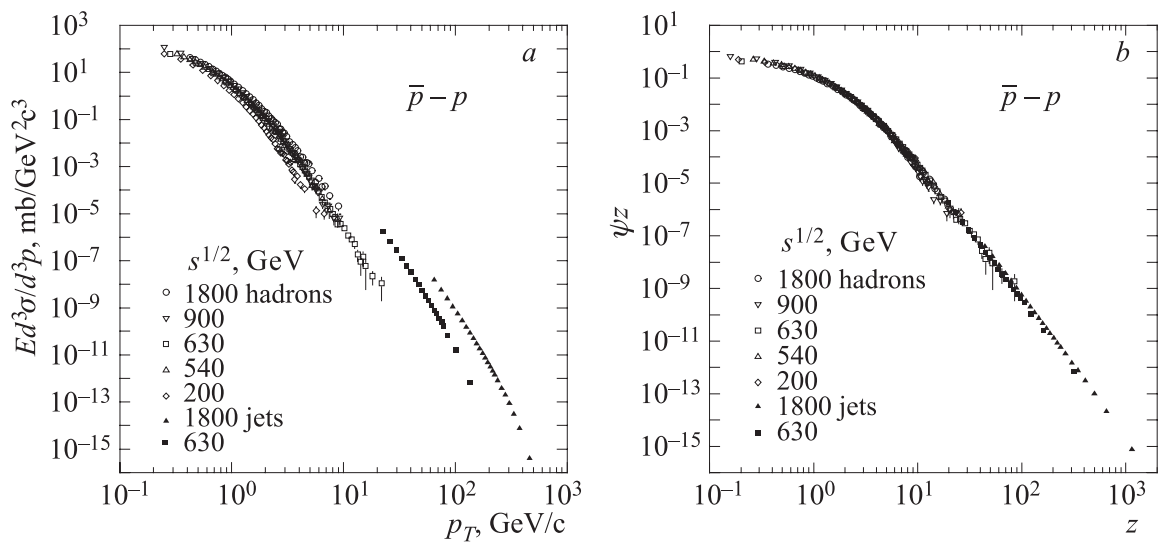

Fig. 5. a) Charged hadron and jet spectra in minimum bias $\bar{p} p$ collisions as a function of collision energy. Experimental data are taken from [20] and [21]. b) The corresponding scaling function $\psi(z)$

and $1800 \mathrm{GeV}$ are plotted in the same figure. Note the strong dependence of cross sections on the collision energy which increases with transverse momentum. The large values of transverse momenta up to $p_{T}=450 \mathrm{GeV} / \mathrm{c}$ correspond to interactions at very small scales. 
It is possible to obtain the $z$-presentation of the charged hadron and jet spectra with the same scaling function $\psi(z)$ shown in Fig. 5, $b$. The values of $\psi$ vary over 15 orders of magnitude corresponding to the range $z=0.2-1000$. We observe well matching of the scaling function for charged hadrons and jets in large overlapping region. The shape of the scaling curve is different in low- and high- $z$ region. The first one is characteristic for soft regime of particle production. The power behavior, $\psi(z) \sim z^{-\beta}$, seen for $z>4$ is typical for hard processes. The common description was obtained for different values of fractal anomalous dimension $\epsilon$ and parameter $c$ for both classes of events. We found $\epsilon=1, c=0.25$ for charged hadrons and $\epsilon=0, c=1$ for jets. The fractal dimensions $\delta_{1}$ and $\delta_{2}$ are equal to 1 in both cases.

Let us discuss the obtained values of the parameters $\epsilon$ and $c$. The zero value of $\epsilon$ for jet production means that all momentum of the outgoing constituent is carried out by jet $(y=1)$. In the case of inclusive hadron production, only part of the constituent momentum $(y<1)$ is transferred to the detected particle. This is reflected by the nonzero anomalous dimension $\epsilon=1$. Results of our analysis show jump of the «heat capacity» $c$ from 0.25 for charged hadrons to 1 for jets. This reflects transition from hadron to quark and gluon degrees of freedom. Strong correlation between the «heat capacity» and the anomalous fractal dimension $\epsilon$ is observed. Final states, hadron and parton $\equiv j e t$, characterized by these parameters, are different for the two classes of events.

\section{CONCLUSIONS}

Dependence of charged hadron spectra on multiplicity density measured by the UA1, E735 and CDF Collaborations in $\bar{p} p$ collisions was studied in the framework of the $z$-scaling concept. $z$-presentation of the data reveals basic features established in minimum bias events. We showed that beside energy independence, the scaling function $\psi(z)$ indicates multiplicity independence in the whole measured $d N_{\mathrm{ch}} / d \eta$ range. New experimental data on multiplicity dependencies of charged hadron spectra in $p p$ collisions obtained by the STAR Collaboration at RHIC additionally confirm the observed results.

Generalized scaling for the inclusive charged hadron and jet production was suggested. It was shown that the same scaling function $\psi(z)$ expressed via the invariant inclusive cross section $E d^{3} \sigma / d p^{3}$ and the charged hadron multiplicity density $d N /\left.d \eta\right|_{0}$ describes both classes of events. The variable $z$ has property of a fractal measure connected with parton content of the composite structures involved. The constituent subprocess underlying production of the inclusive particle/jet is determined by minimal resolution of the fractal measure $z$ with respect to all subprocesses in which the inclusive particle/jet can be produced.

The anomalous fractal dimensions and the parameter $c$ for charged hadrons and jets were found to be $\delta_{1}=\delta_{2}=1, \epsilon=1, c=0.25$ and $\delta_{1}=\delta_{2}=1, \epsilon=0$, 
$c=1$, respectively. Connection between the scaling variable and entropy was used to interpret the parameter $c$ as «heat capacity» of the produced medium and the multiplicity density in the central region $d N /\left.d \eta\right|_{0}$ as «temperature» of the colliding system. Analysis of experimental data on charged particle production in $p \bar{p}$ and $p p$ collisions indicates constancy of the «heat capacity» $c$ for various energies and multiplicities. Jump of the «heat capacity» from 0.25 for charged hadrons to 1 for jets was argued as indication of phase transition from hadron to quark-gluon degrees of freedom.

The obtained results are of interest for searching and study of new physics phenomena in particle production over a wide range of collision energies and multiplicity densities at the U70, Tevatron, RHIC and LHC.

Acknowledgments. The authors would like to thank Yu. Panebratsev for his support of this work. The investigation has been partially supported by the IRP AVOZ10480505 and by the Grant Agency of the Czech Republic under the contract No. 202/04/0793.

\section{REFERENCES}

1. Eichten E., Lane K., Peskin M. // Phys. Rev. Lett. 1983. V.50. P.811;

Eichten E., Hinchliffe I., Lane K., Quigg C. // Rev. Mod. Phys. 1984. V. 56. V.4.

2. Antoniadis I. // Proc. of European School of High-Energy Physics, Beatenberg, Switzerland, 26 August-8 September, 2001. P. 301.

3. Lester C. G. // Proc. of Advanced Studies Institute on Physics at LHC, Prague, July 6-12, 2003. P. A303.

4. Nottale L. Fractal Space-Time and Microphysics. Singapore: World Sci., 1993; Mandelbrot B. The Fractal Geometry of Nature. San Francisco: Freeman, 1982.

5. Feynman R. P. // Phys. Rev. Lett. 1969. V.23. P. 1415.

6. Bjorken J. D. // Phys. Rev. 1969. V. 179. P. 1547;

Bjorken J. D., Paschanos E. A. // Phys. Rev. 1969. V. 185. P. 1975.

7. Bosted P. et al. // Phys. Rev. Lett. 1972. V.49. P. 1380.

8. Benecke J. et al. // Phys. Rev. 1969. V.188. P. 2159.

9. Baldin A. M. // Sov. J. Part. Nucl. 1977. V.8. P. 429.

10. Stavinsky V. S. // Sov. J. Part. Nucl. 1979. V. 10. P. 949.

11. Leksin G. A. Report No. ITEF-147, 1976;

Leksin G. A. // Proc. of the XVIII International Conference on High Energy Physics, Tbilisi, Georgia, 1976. D1,2-10400. Tbilisi, 1977. P. A6-3. 
12. Koba Z., Nielsen H. B., Olesen P. // Nucl. Phys. B. 1972. V.40. P. 317.

13. Matveev V.A., Muradyan R.M., Tavkhelidze A.N. // Part. Nuclei. 1971. V.2. P.7; Lett. Nuovo Cim. 1972. V. 5. P.907; Lett. Nuovo Cim. 1973. V. 7. P. 719.

14. Brodsky S., Farrar G. // Phys. Rev. Lett. 1973. V.31. P. 1153; Phys. Rev. D. 1975. V.11. P. 1309.

15. Zborovský I., Panebratsev Yu.A., Tokarev M. V., Škoro G. P. // Phys. Rev. D. 1996. V.54. P. 5548;

Zborovský I., Tokarev M. V., Panebratsev Yu.A., Škoro G. P. // Phys. Rev. C. 1999. V.59. P. 2227;

Tokarev M. V., Dedovich T. G. // Int. J. Mod. Phys. A. 2000. V. 15. P. 3495;

Tokarev M. V., Rogachevski O.V., Dedovich T.G. // J. Phys. G: Nucl. Part. Phys. 2000. V 26. P. 1671;

Tokarev M. V., Rogachevski O. V., Dedovich T. G. JINR Preprint E2-2000-90. Dubna, 2000;

Tokarev M., Zborovský I., Panebratsev Yu., Skoro G. // Int. J. Mod. Phys. A. 2001. V.16. P. 1281;

Tokarev M. hep-ph/0111202;

Tokarev M., Toivonen D. hep-ph/0209069;

Skoro G. P., Tokarev M. V., Panebratsev Yu. A., Zborovský I. hep-ph/0209071;

Tokarev M. V., Efimov G.L., Toivonen D.E. // Phys. Atom. Nucl. 2004. V.67. P.564; Tokarev M. // Acta Phys. Slov. 2004. V.54. P. 321;

Tokarev M. V., Dedovich T. G. // Phys. Atom. Nucl. 2005. V.68. P. 404.

16. Arnison G. et al. // Phys. Lett. B. 1982. V. 118. P. 167.

17. Alexopoulos T. et al. // Phys. Lett. B. 1994. V.336. P. 599.

18. Acosta D. et al. // Phys. Rev. D. 2002. V.65. P. 072005.

19. Gans J.E. PhD Thesis. Yale University, USA, 2004.

20. Arnison G. et al. // Phys. Lett. B. 1982. V. 118. P. 167; Abe F. et al. // Phys. Rev. Lett. 1988. V.61. P. 1819; Albajar C. et al. // Nucl. Phys. B. 1990. V.335. P.261; Bocquet G. et al. // Phys. Lett. B. 1996. V.366. P.434;

21. Abbott B. et al. // Phys. Rev. D. 2001. V.64. P. 032001.

Received on June 1, 2005. 
Корректор T. Е. Попеко

Подписано в печать 20.07.2005.

Формат $60 \times 90 / 16$. Бумага офсетная. Печать офсетная.

Усл. печ. л. 1,18. Уч.-изд. л. 1,67. Тираж 415 экз. Заказ № 54968.

Издательский отдел Объединенного института ядерных исследований 141980, г. Дубна, Московская обл., ул. Жолио-Кюри, 6.

E-mail: publish@pds.jinr.ru www.jinr.ru/publish/ 